\title{
FINITE HJELMSLEV PLANES WITH NEW INTEGER INVARIANTS
}

\author{
BY DAVID A. DRAKE ${ }^{1}$ AND HANFRIED LENZ \\ Communicated March 28, 1975
}

Projective Hjelmslev planes (PH-planes) are a generalization of projective planes in which each point-pair is joined by at least one line and, dually, each line-pair has a nontrivial intersection. Multiply joined points (and multiply intersecting lines) are called neighbor points (and neighbor lines). By hypothesis, the neighbor relations of a PH-plane $A$ are equivalence relations which induce a canonical epimorphism from $A$ to a projective plane $\bar{A}$. If $A$ is finite, there exists [4] an integer $t$ such that the inverse image of every point and every line of $\bar{A}$ contains precisely $t^{2}$ elements. If the order of $\bar{A}$ is $r$, we say that $A$ is a $(t, r)$ PH-plane. We are concerned with the problem of determining the spectrum $S$ of all admissible pairs $(t, r)$. Since the finite projective planes are simply the $(1, r)$ PH-planes, our concern is with a generalization of the classical existence question for projective planes.

Prior to this announcement, the only pairs $(t, r)$ known to belong to $S$ satisfy the requirements:

(1) $t$ is a power of $r$,

(2) $r$ is a prime power.

Conversely, all such pairs do belong to $S$, and all arise as the invariants of the Desarguesian-Pappian PH-planes investigated by Klingenberg [5] . A deep theorem of Artmann [1] allows one to assert that $(t, r)$ is in $S$ if (1) holds and if $r$ is the order of a projective plane. Whether this is any improvement over the previous result is, however, still uncertain.

Nonexistence results to date are also few in number. The celebrated Bruck-Ryser Theorem gives infinitely many values of $r$ for which $(1, r) \notin S$. Clearly $(1, r) \notin S$ implies $(t, r) \notin S$ for any $t$. Kleinfield [4] has observed that $(t, r) \in S$ with $t \neq 1$ implies $t \geqslant r$. Most recently, Drake [2] has proved that $(t, r) \in S$ with $1 \neq t \neq r$ implies that $t=4$ or 8 or that $r \leqslant t+1-\sqrt{ }(2 t+3)$.

The current note is written to announce the following two existence results:

AMS (MOS) subject classifications (1970). Primary 05B25; Secondary 05B20.

Key words and phrases. Projective Hjelmslev plane, projective plane, permutation and incidence matrices, Desarguesian, Pappian.

${ }^{1}$ Supported by NSF Grant GP-39059, a University of Florida Faculty Development Grant and an Alexander von Humboldt Dozentenstipendium. 
TheOREM 1. Let $t, r, q, b$ be positive integers such that $(t, r) \in S, q$ is $a$ prime power and $q+1=t(r+1)$. Then $\left(t \cdot q^{b}, r\right) \in S$.

Theorem 2. Let $t, q, b$ be positive integers such that $(t, t) \in S, q$ is a prime power and $2(t+1) \leqslant q+1 \leqslant t(t+1)$. Then $\left(t \cdot q^{b}, t\right) \in S$.

Details will be given elsewhere of a construction which simultaneously yields both theorems and a little more. Theorem 1, for example, allows one to conclude that $\left(8 \cdot 23^{b}, 2\right) \in S$ for arbitrary $b$. The actual Lenz-Drake construction applied to the "extremal" $(8,2)$ PH-planes of Shult and Drake [3] yields the additional information that $\left(8 \cdot 19^{b}, 2\right),\left(8 \cdot 17^{b}, 2\right) \in S$.

We remark that Theorem 1 may be applied either recursively or in tandem with Theorem 2. For example, since $(2,2) \in S$, Theorem 1 (or 2) yields $\left(2 \cdot 5^{b}, 2\right) \in S$. A second application of Theorem 1 then yields $\left(2 \cdot 5 \cdot 29^{d}, 2\right)$, $\left(2 \cdot 25 \cdot 149^{d}, 2\right) \in S$.

The construction is largely elementary. We mention several of the basic ideas, presenting them in generality sufficient for the proof of Theorem 1 . If $M=\left[m_{i j}\right]$ is an incidence matrix for a $(t, r)$ PH-plane $A$, then every row and every column of $M$ contains precisely $t(r+1)$ one's. Thus König's Lemma implies that $M$ is a sum of permutation matrices; consequently, it is possible to obtain a matrix $N=\left[n_{i j}\right]$ of the same size as $M$ such that $n_{i j}=0$ precisely when $m_{i j}=0$ and so that every integer from 1 to $t(r+1)$ appears in each row and each column of $N$. Next one seeks a suitable set of $t(r+1)$ square matrices $B_{1}$, $B_{2}, \ldots$ of order $s^{2}$ where $s=q^{b}$. One then obtains a matrix $G$ from $N$ by substituting $B_{i}$ for $i$ when $i \geqslant 1$ and replacing each 0 by the square zero matrix of order $s^{2}$. For $G$ to represent the desired $(t \cdot s, r)$ PH-plane, it suffices to demand that the matrices $B_{i}$ satisfy:

$$
B_{i} \cdot\left(B_{j}\right)^{T}=\left(B_{j}\right)^{T} \cdot B_{i}=J \quad \text { when } i \neq j
$$

and

$$
\sum B_{i} \cdot\left(B_{i}\right)^{T}, \quad \sum\left(B_{i}\right)^{T} \cdot B_{i} \geqslant 2 J
$$

here $J$ denotes the matrix of all one's, and one writes $\left[x_{i j}\right] \geqslant\left[y_{i j}\right]$ to mean that $x_{i j} \geqslant y_{i j}$ for all $i, j$.

The $B_{i}$ can be obtained from an $(s, q)$ PH-plane $A^{\prime}$ of the type investigated by Klingenberg and mentioned above. One obtains an incidence matrix $D=$ $\left[D_{i j}\right]$ for $A^{\prime}$ so written that every $D_{i j}$ is square of order $s^{2}$ and successive sets of $s^{2}$ columns (rows) represent neighbor classes of lines (points). Let $E_{1}, E_{2}, \ldots$ be the nonzero matrices among the $D_{1 x} ; F_{1}, F_{2}, \ldots$ be the nonzero matrices a among the $D_{x 1}$. Then there exist permutation matrices $P_{i}, Q_{i}$ such that $E_{i} P_{i}=$ $Q_{i} F_{i} \equiv B_{i}$ for all $i$, and these $B_{i}$ satisfy conditions (3) and (4). 


\section{REFERENCES}

1. B. Artmann, Existenz und projektive Limiten von Hjelmslev-Ebenen $n$-ter Stufe, Atti del Convergno di geometria Combinatoria e sue Applicazioni (Univ. degli Studi di

Perugia, Perugia, 1970), Int. Mat., Univ. Perugia, Perugia, 1971, pp. 27-41. MR 49 \#7910.

2. D. Drake, Nonexistence results for finite Hjelmslev planes, Abh. Math. Sem. Univ. Hamburg 40 (1974), 100-110. MR 49 \#3676.

3. D. Drake and E. Shult, Extremal Hjelmslev planes (to appear).

4. E. Kleinfeld, Finite Hjelmslev planes, Illinois J. Math. 3 (1959), 403-407. MR 21 \#5934.

5. W. Klingenberg, Projektive und affine Ebenen mit Nachbarelementen, Math. Z. 60 (1954), 384-406. MR 16, 507.

DEPARTMENT OF MATHEMATICS, UNIVERSITY OF FLORIDA, GAINESVILLE, FLORIDA 32611 (Current address of David A. Drake)

FACHBEREICH MATHEMATIK DER FREIEN UNIVERSITÄT, 1 BERLIN 33, GERMANY (Current address of Hanfried Lenz) 